\title{
Stability analysis of the generalized fractional differential equations with and without exogenous inputs
}

\section{Ndolane Sene}

Laboratoire Lmdan, Département de Mathématiques de la Décision, Université Cheikh Anta Diop de Dakar, BP 5683 Dakar Fann, Senegal.

\begin{abstract}
The stability conditions of the fractional differential equations described by the Caputo generalized fractional derivative have been addressed. The generalized asymptotic stability of a class of the fractional differential equations has been investigated. The fractional input stability in the context of the fractional differential equations described by the Caputo generalized fractional derivative has been introduced. The Lyapunov characterizations of the generalized asymptotic stability and the generalized fractional input stability of the fractional differential equations with or without inputs have been provided. Several examples illustrating the main results of the paper have been proposed. The Caputo generalized fractional derivative and the generalized Gronwall lemma have been used.
\end{abstract}

Keywords: Caputo generalized fractional derivative, asymptotic stability, fractional differential equations.

2010 MSC: 93D05, 26A33, 93D25.

(C)2019 All rights reserved.

\section{Introduction}

Recently, the fractional calculus has received increasing attention due to its applications in many fields as: synchronization, tracking controller, physics, control engineering, signal processing, complex systems $[4,25]$. Various types of the fractional derivative operators exist in the literature as: the Riemann-Liouville derivative [13, 14], the Caputo derivative [16, 21], the Atangana-Baleanu derivative[2], the Atangana Koca derivative [3], the Conformable derivative [23], the generalized fractional derivative [1, 12], and others.

In this paper, we use the Caputo generalized fractional derivative introduced by Udita in [11, 12]. We will use the Caputo generalized fractional derivative of analyzing the stability and the generalized fractional input stability of the fractional differential equations with or without inputs. In these last five years, Thabet and Fahd have done many investigations related to certain generalized fractional derivative operators. They have established many results $[1,5,9,10]$. In $[1,24]$, Fahd et al. have introduced a new version of the generalized Gronwall lemma. This lemma has many applications in this paper. The generalized Gronwall lemma will be used in this paper of getting the conditions of the global asymptotic stability of the trivial solution of the perturbed generalized fractional differential equations. In numerous

Email address: ndolanesene@yahoo.fr (Ndolane Sene)

doi: $10.22436 /$ jnsa.012.09.01

Received: 2018-09-22 Revised: 2019-02-14 Accepted: 2019-02-28 
cases proving the generalized asymptotic stability condition and the generalized fractional input stability using the trajectories are not trivial. Therefore, we propose an alternative consisting of using the Lyapunov characterizations of addressing these issues.

The remainder of this paper is organized as follows. In Section 2, we introduce specific basic definitions and lemmas. We will use them to analyze the stability notions of the fractional differential equations described by the Caputo generalized fractional derivative. In Section 3, we study the generalized asymptotic stability of the trivial solution of the fractional differential equations using the Lyapunov direct method. In Section 4, we analyze the generalized asymptotic stability of the trivial solution of the perturbed fractional differential equations. In Section 5, we introduce the generalized fractional input stability of the fractional differential equations with exogenous inputs. In Section 6, we provided numerical examples of illustrating our main results.

\section{Preliminary definitions and results}

In this section, we introduce specific notations and preliminary definitions. We will use the definitions and the notions to establish the main results. We begin by adding the comparison functions. These functions are fundamental in the stability analysis, notably in the application of the Lyapunov direct method.

$\mathcal{P D}$ denotes the set of all continuous functions $\alpha: \mathbb{R}_{\geqslant 0} \rightarrow \mathbb{R}_{\geqslant 0}$ satisfying $\alpha(0)=0$, and $\alpha(s)>0$ for all $s>0$. A class $\mathcal{K}$ function is an increasing $\mathcal{P D}$ function.

The class $\mathcal{K}_{\infty}$ represents the set of all unbounded $\mathcal{K}$ functions. A continuous function $\beta: \mathbb{R}_{\geqslant 0} \times \mathbb{R}_{\geqslant 0} \rightarrow$ $\mathbb{R}_{\geqslant 0}$ is said to be of class $\mathcal{K} \mathcal{L}$ if $\beta(., t) \in \mathcal{K}$ for any $t \geqslant 0$, and $\beta(s,$.$) is non-increasing and tends to zero as$ its arguments tend to infinity.

We begin this section by recalling the definitions of the left generalized fractional derivative, the Caputo generalized fractional derivative and the generalized fractional integral.

Definition $2.1([1,11])$. The left generalized fractional derivative of order $\alpha \in[0,1), \rho>0$ of a continuous function $f:[a,+\infty[\longrightarrow \mathbb{R}$ is given by

$$
\left(D^{\alpha, \rho_{f}}\right)(t)=\frac{1}{\Gamma(1-\alpha)}\left(t^{1-\rho} \frac{d}{d t}\right) \int_{a}^{t}\left(\frac{t^{\rho}-s^{\rho}}{\rho}\right)^{-\alpha} f(s) \frac{d s}{s^{1-\rho}}
$$

for all $t>a$. The function $\Gamma($.$) denotes the Gamma function.$

Definition $2.2([1,11])$. The generalized fractional integral of order $\alpha \in[0,1), \rho>0$ of a continuous function $f:[a,+\infty[\longrightarrow \mathbb{R}$ is given by

$$
\left(I^{\alpha, \rho} f\right)(t)=\frac{\rho^{1-\alpha}}{\Gamma(\alpha)} \int_{a}^{t}\left(t^{\rho}-s^{\rho}\right)^{\alpha-1} f(s) \frac{d s}{s^{1-\rho}}
$$

for all $t>a$. The function $\Gamma($.$) denotes the Gamma function.$

Definition $2.3([1,10,11])$. The Caputo generalized fractional derivative of order $\alpha \in[0,1), \rho>0$ of a continuous function $f:[a,+\infty[\longrightarrow \mathbb{R}$ is given by

$$
\left(D_{c}^{\alpha, \rho} f\right)(t)=\frac{1}{\Gamma(1-\alpha)} \int_{a}^{t}\left(\frac{t^{\rho}-s^{\rho}}{\rho}\right)^{-\alpha} f^{\prime}(s) d s
$$

for all $t>a$. The function $\Gamma($.$) denotes the Gamma function.$

We can observe that if $\rho \rightarrow 0$ in Definition 2.1, we recover the Riemann-Liouville fractional derivative as defined in $[13,14]$. Let's recall the definition of the Mittag-Leffler function. This function has many applications in the fractional calculus. For example, they play an important role in the structure of the analytical solution of the Hristov diffusion equations [17]. 
Definition 2.4 ([1, 15]). Let $\alpha>0, \beta \in \mathbb{R}$ and $z \in \mathbb{C}$. The Mittag-Leffler function is defined by the series

$$
\mathrm{E}_{\alpha, \beta}(z)=\sum_{k=0}^{\infty} \frac{z^{\mathrm{k}}}{\Gamma(\alpha \mathrm{k}+\beta)}, \quad \mathrm{E}_{\alpha}(z)=\mathrm{E}_{\alpha, 1}(z) \quad \mathrm{E}_{1}(z)=\exp (z) .
$$

When $\alpha>0$ and $\beta>0$, the series is convergent.

In numerous cases, when we want to calculate the Caputo generalized fractional derivative of a Lyapunov function, it is not trivial. We give the following lemma. It will help us of studying the stability notions using the Lyapunov direct methods. We adopt the same method as in [7].

Lemma 2.5. Let $x \in \mathbb{R}^{n}$ be a vector of differentiable functions. Then, for any time $t \geqslant 0$, the following relationship holds

$$
\mathrm{D}_{\mathrm{c}}^{\alpha, \rho}\left(\mathrm{x}^{\top} \mathrm{P} x\right) \leqslant 2 x^{\top} \mathrm{PD}_{\mathrm{c}}^{\alpha, \rho} x, \quad \alpha \in[0,1), \rho>0,
$$

where $\mathrm{P} \in \mathbb{R}^{\mathrm{n} \times \mathrm{n}}$ is a constant, square, symmetric, and positive definite matrix.

Proof. Let's the function $f(t)=D_{c}^{\alpha, \rho}\left(x^{\top} P x\right)-2 x^{\top} P D_{c}^{\alpha, \rho} \chi$. We will prove the function $f$ is negative definite. We rewrite the function $f$ using the definition of the Caputo generalized fractional derivative, and we obtain that:

$$
\begin{aligned}
f(t) & =\frac{1}{\Gamma(1-\alpha)} \int_{t_{0}}^{t}\left(\dot{x}^{\top}(s) P x(s)-x^{\top}(s) P \dot{x}(s)\right)\left(\frac{t^{\rho}-s^{\rho}}{\rho}\right)^{-\alpha} d s-\frac{2 x^{\top}(t) P}{\Gamma(1-\alpha)} \int_{t_{0}}^{t} \dot{x}(s)\left(\frac{t^{\rho}-s^{\rho}}{\rho}\right)^{-\alpha} d s \\
& =\frac{1}{\Gamma(1-\alpha)} \int_{t_{0}}^{t}\left(\dot{x}^{\top}(s) P x(s)-x^{\top}(s) P \dot{x}(s)-2 x^{\top}(t) P \dot{x}(s)\right)\left(\frac{t^{\rho}-s^{\rho}}{\rho}\right)^{-\alpha} d s \\
& =\frac{1}{\Gamma(1-\alpha)} \int_{t_{0}}^{t}\left(2 x^{\top}(s) P \dot{x}(s)-2 x^{\top}(t) P \dot{x}(s)\right)\left(\frac{t^{\rho}-s^{\rho}}{\rho}\right)^{-\alpha} d s .
\end{aligned}
$$

Let's that $y(s)=x^{\top}(s)-x^{\top}(t)$, then the above function $f$ can be rewritten as follows

$$
\begin{aligned}
f(t) & =\frac{1}{\Gamma(1-\alpha)} \int_{t_{0}}^{t} 2 y^{\top}(s) P \dot{y}(s)\left(\frac{t^{\rho}-s^{\rho}}{\rho}\right)^{-\alpha} d s \\
& =\frac{\rho^{\alpha}}{\Gamma(1-\alpha)} \int_{t_{0}}^{t} \frac{2 y^{\top}(s) P \dot{y}(s)}{\left(t^{\rho}-s^{\rho}\right)^{\alpha}} d s=\frac{\rho^{\alpha}}{\Gamma(1-\alpha)} \int_{t_{0}}^{t} \frac{d\left(y^{\top}(s) P y(s)\right)}{\left(t^{\rho}-s^{\rho}\right)^{\alpha}} d s .
\end{aligned}
$$

Using integration by parts, we obtain the following expression

$$
\begin{aligned}
f(t) & =\left[\frac{y^{\top}(s) P y(s)}{\left(t^{\rho}-s^{\rho}\right)^{\alpha}}\right]_{t_{0}}^{t}-\frac{\rho^{\alpha}}{\Gamma(1-\alpha)} \int_{t_{0}}^{t} \frac{\alpha s^{\rho-1} y^{\top}(s) P y(s)}{\left(t^{\rho}-s^{\rho}\right)^{\alpha}} d s \\
& =\lim _{s \rightarrow t} \frac{y^{\top}(s) P y(s)}{\left(t^{\rho}-s^{\rho}\right)^{\alpha}}-\frac{y^{\top}\left(t_{0}\right) P y\left(t_{0}\right)}{\left(t^{\rho}-s^{\rho}\right)^{\alpha}}-\frac{\rho^{\alpha}}{\Gamma(1-\alpha)} \int_{t_{0}}^{t} \frac{\alpha s^{\rho-1} y^{\top}(s) P y(s)}{\left(t^{\rho}-s^{\rho}\right)^{\alpha}} d s .
\end{aligned}
$$

Calculating the limit using the Hospital's rule, we have

$$
\lim _{s \rightarrow t} \frac{y^{\top}(s) P y(s)}{\left(t^{\rho}-s^{\rho}\right)^{\alpha}}=\frac{2\left(x^{\top}(s)-x^{\top}(t)\right) P \dot{x}(s)\left(t^{\rho}-s^{\rho}\right)^{1-\alpha}}{\rho s^{\rho-1}}=0 .
$$

The function $\mathrm{f}$ becomes the following

$$
f(t)=-\frac{y^{\top}\left(t_{0}\right) P y\left(t_{0}\right)}{\left(t^{\rho}-s^{\rho}\right)^{\alpha}}-\frac{\rho^{\alpha}}{\Gamma(1-\alpha)} \int_{t_{0}}^{t} \frac{\alpha s^{\rho-1} y^{\top}(s) P y(s)}{\left(t^{\rho}-s^{\rho}\right)^{\alpha}} d s .
$$

Finally, we observe that $f(t)=D_{c}^{\alpha, \rho}\left(x^{\top} P x\right)-2 x^{\top} P D_{c}^{\alpha, \rho} x \leqslant 0$, from which we obtain $D_{c}^{\alpha, \rho}\left(x^{\top} P x\right) \leqslant$ $2 x^{\top} \operatorname{PD}_{c}^{\alpha, \rho} x$ for all $\alpha \in[0,1)$ and $\rho>0$. 
The Cauchy problem has an essential role in the resolution of several differential fractional equations. To this end, we make the following lemma.

Lemma 2.6. The solution of the Cauchy problem defined by $D_{c}^{\alpha, \rho} x(t)=\lambda x(t)$ with initial boundary condition $x(0)=x_{0}=\eta$ is given by

$$
x(t)=\eta E_{\alpha}\left(\lambda\left(\frac{t^{\rho}-t_{0}^{\rho}}{\rho}\right)^{\alpha}\right) .
$$

Proof. We apply the $\rho$-Laplace transform $\mathcal{L}_{\rho}$ [8] to both sides of equation $D_{c}^{\alpha, \rho} x(t)=\lambda x(t)$. We have

$$
\mathcal{L}_{\rho}\left(D_{c}^{\alpha, \rho} \chi(t)\right)=\lambda \mathcal{L}_{\rho}(x(t)), \quad s^{\alpha} \mathcal{L}_{\rho}(x(t))-s^{\alpha-1} \eta=\lambda \mathcal{L}_{\rho}(x(t)), \quad \mathcal{L}_{\rho}(x(t))=\frac{s^{\alpha-1} \eta}{s^{\alpha}-\lambda} .
$$

Applying the inverse of $\rho$-Laplace transform to both sides of equation (2.1) and using Lemma 3.4 in [8], we obtain

$$
x(t)=\eta E_{\alpha}\left(\lambda\left(\frac{t^{\rho}-t_{0}^{\rho}}{\rho}\right)^{\alpha}\right) .
$$

The solution of the fractional differential equation defined by $D^{\alpha, \rho} \chi(t)=\lambda x(t)$ can be found in [1]. We give in the following lemma, the solution of the fractional differential equation with perturbation term.

Lemma 2.7 ([8]). The generalized fractional differential equation defined by $D_{c}^{\alpha, \rho} x(t)=\lambda x(t)+m(t)$ with initial condition $x(0)=x\left(t_{0}\right)=\eta$ is given by

$$
x(t)=\eta E_{\alpha}\left(\lambda\left(\frac{t^{\rho}-t_{0}^{\rho}}{\rho}\right)^{\alpha}\right)+\int_{t_{0}}^{t}\left(\frac{t^{\rho}-s^{\rho}}{\rho}\right)^{\alpha-1} E_{\alpha, \alpha}\left(\lambda\left(\frac{t^{\rho}-t_{0}^{\rho}}{\rho}\right)^{\alpha}\right) m(s) \frac{d s}{s^{1-\rho}} .
$$

The proof of Lemma 2.7 can be found in [8]. The Lemmas 2.6 and 2.7 play a fundamental role in the problem consisting of getting the analytical solutions of the generalized fractional differential equations. We recall the generalized Gronwall lemma inequality, seen in [1] for more information.

Lemma $2.8([1])$. Let $\alpha>0, x(t), a(t)$ be non negative function and $b(t)$ be non-negative and non decreasing function for $\mathrm{t} \in\left[\mathrm{t}_{0}, \mathrm{~T}\right], \mathrm{T}>0, \mathrm{~b}(\mathrm{t}) \leqslant \mathrm{M}$ where $\mathrm{M}$ is constant. If the following inequality is hold

$$
x(t) \leqslant a(t)+b(t) \int_{t_{0}}^{t}\left(\frac{t^{\rho}-s^{\rho}}{\rho}\right)^{\alpha-1} x(s) \frac{d s}{s^{1-\rho}}
$$

then we obtain the inequality defined by

$$
x(t) \leqslant a(t)+\int_{t_{0}}^{t} \sum_{n=1}^{\infty} \frac{b(t) \Gamma(\alpha)}{\Gamma(n \alpha)}\left(\frac{t^{\rho}-s^{\rho}}{\rho}\right)^{n \alpha-1} a(s) \frac{d s}{s^{1-\rho}}
$$

with $\mathrm{t} \in\left[\mathrm{t}_{0}, \mathrm{~T}\right]$. Assume furthermore that $\mathrm{a}(\mathrm{t})$ is non decreasing for $\mathrm{t} \in\left[\mathrm{t}_{0}, \mathrm{~T}\right]$. Then we have the following inequality

$$
x(t) \leqslant a(t) E_{\alpha}\left(b(t) \Gamma(\alpha)\left(\frac{t^{\rho}-t_{0}^{\rho}}{\rho}\right)\right) .
$$

With the generalized Gronwall lemma, we can also recover the particular Gronwall lemma as defined in [16]. 


\section{Stability analysis of the generalized fractional differential equations}

The generalized fractional differential equation under consideration is represented by the following equation

$$
D_{c}^{\alpha, \rho} x(t)=f(t, x),
$$

where $x \in \mathbb{R}^{n}$ is a state variable, and $f: \mathbb{R}^{+} \times \mathbb{R}^{n} \rightarrow \mathbb{R}^{n}$ is a continuous locally Lipschitz function and admits zero as an equilibrium point. In other words, we have $f(t, 0)=0$. Given an initial condition $x_{0} \in \mathbb{R}^{n}$, the solution of (3.1) starting at $x_{0}$ at time $t_{0}$ is denoted by $x()=.x\left(., x_{0}\right)$. For the rest of the paper we suppose the initial condition $x_{0}=\eta$.

Based on the fact that the function $f$ is continuous and locally Lipschitz, the solution of the generalized fractional differential equation defined by (3.1) exists at all forward times. This remark is necessary for the rest of the paper. We recall some definitions related to the stability notions. We have the following definitions.

Definition 3.1. The origin of the generalized fractional differential equation defined by (3.1) is said to be generalized Mittag-Leffler stable if, for any initial condition $\eta$, its solution satisfies

$$
\left\|x\left(t, x_{0}\right)\right\| \leqslant\left[m(\|\eta\|) E_{\alpha}\left(\lambda\left(\frac{t^{\rho}-t_{0}^{\rho}}{\rho}\right)^{\alpha}\right)\right]^{b},
$$

where $b>0, \lambda<0$, and $m$ is locally Lipschitz on a domain contained in $\mathbb{R}^{n}$ with a Lipschitz constant $K$ and satisfies $m(0)=0$.

We give the definition of the generalized global asymptotic stability. Note the class $\mathcal{K} \mathcal{L}$ function is proportional to the quantity $\mathrm{t}^{\rho}-\mathrm{t}_{0}^{\rho}$.

Definition 3.2. The generalized fractional differential equation defined by (3.1) is said to be generalized globally asymptotically stable if there exist a class $\mathcal{K} \mathcal{L}$ function $\beta$ such that for any initial condition $\eta$, the following inequality holds

$$
\left\|x\left(t, x_{0}\right)\right\| \leqslant \beta\left(\|\eta\|, t^{\rho}-t_{0}^{\rho}\right) .
$$

We introduce the first primary result of this paper. We extend the Lyapunov direct method to the stability analysis of the trivial solution of the fractional differential equations described by the Caputo fractional derivative. We have the following theorem.

Theorem 3.3. Let there exists a positive function $\mathrm{V}: \mathbb{R}^{+} \times \mathbb{R}^{\mathrm{n}} \longrightarrow \mathbb{R}$ be continuous, and class $\mathcal{K}_{\infty}$ functions $\chi_{2}, \chi_{3}, \chi_{4}$ satisfying the following conditions

1. $\chi_{2}(\|x\|) \leqslant V(t, x) \leqslant \chi_{3}(\|x\|)$;

2. $\mathrm{V}(\mathrm{t}, \mathrm{x})$ has the Caputo generalized fractional derivative of order $\alpha, \rho$ for $\mathrm{t}>\mathrm{t}_{0} \geqslant 0$;

3. $\mathrm{D}_{\mathrm{c}}^{\alpha, \rho} \mathrm{V}(\mathrm{t}, \mathrm{x}) \leqslant-\chi_{4}(\|x\|)$,

then $x=0$ of the generalized fractional differential equation (3.1) is generalized globally uniformly asymptotically stable.

Note that the first assumption is equivalent to the function $V$ is radially unbounded. In other words, the function $V$ is a Lyapunov candidate function.

Proof. Combining the first and the second assumptions, we get the following inequality

$$
\mathrm{D}_{\mathrm{c}}^{\alpha, \rho} \mathrm{V}(\mathrm{t}, \mathrm{x}) \leqslant-\chi_{4}\left(\chi_{3}^{-1}(\mathrm{~V}(\mathrm{t}, \mathrm{x}))=-\mathrm{g}(\mathrm{V}(\mathrm{t}, \mathrm{x}) .\right.
$$

Let $y(t)$ be the solution of the fractional differential equation defined by $D_{c}^{\alpha, \rho} y=-g(y)$, whenever $y\left(t_{0}\right) \geqslant V\left(t_{0}\right)$. Using $\mathcal{K L}$-estimate for the fractional differential equations, see in [15], there exists a class $\mathcal{K} \mathcal{L}$ function $\mu$ such that

$$
V(t, x) \leqslant \mu\left(V\left(x_{0}\right), t^{\rho}-t_{0}^{\rho}\right) .
$$


From first assumption, it yields that $x_{2}(\|x\|) \leqslant \mu\left(V\left(x_{0}\right), t^{\rho}-t_{0}^{\rho}\right)$. From which we obtain the following inequality $\|x(t)\| \leqslant x_{2}^{-1}\left(2 \mu\left(V\left(x_{0}\right), t^{\rho}-t_{0}^{\rho}\right)\right)$. Notice that the function $\left.\beta\left(\left\|x_{0}\right\|, t^{\rho}-t_{0}^{\rho}\right)\right)=x_{2}^{-1}\left(2 \mu\left(V\left(x_{0}\right), t^{\rho}\right.\right.$ $\left.-\mathrm{t}_{0}^{\rho}\right)$ ) is a class $\mathcal{K} \mathcal{L}$ function. Finally, we have the following inequality

$$
\|x(t)\| \leqslant \beta\left(\left\|x_{0}\right\|, t^{\rho}-t_{0}^{\rho}\right) .
$$

Thus, the trivial solution $x=0$ for the generalized fractional differential equation defined by (3.1) is generalized globally uniformly asymptotically stable.

To apply the exponential form of the Lyapunov function [15] for the fractional differential equations, we make the following theorem.

Theorem 3.4. Let there exists a positive function $\mathrm{V}: \mathbb{R}^{+} \times \mathbb{R}^{\mathrm{n}} \longrightarrow \mathbb{R}$ be continuous, and class $\mathcal{K}_{\infty}$ functions $\chi_{2}, \chi_{3}, \chi_{4}$ satisfying the following conditions:

1. $\|\mathrm{x}(\mathrm{t})\|^{\mathrm{a}} \leqslant \mathrm{V}(\mathrm{t}, \mathrm{x}) \leqslant \mathrm{x}_{3}(\|\mathrm{x}\|)$ with a a positive constant;

2. $D_{c}^{\alpha, \rho} V(t, x) \leqslant-\chi_{4}(\|x\|)$,

then $x=0$ of the generalized fractional differential equations (3.1) is generalized Mittag-Leffler stable.

Proof. Similar to the above proof, combining the first and the second assumptions, we get the following inequality

$$
\mathrm{D}_{\mathrm{c}}^{\alpha, \rho} \mathrm{V}(\mathrm{t}, \mathrm{x}) \leqslant-\chi_{4}\left(\chi_{3}^{-1}(\mathrm{~V}(\mathrm{t}, \mathrm{x})) .\right.
$$

Using the exponential form of Lyapunov function for the fractional differential equations [15], there exist a positive constant $k$ such that

$$
\mathrm{D}_{\mathrm{c}}^{\alpha, \rho} \mathrm{V}(\mathrm{t}, \mathrm{x}) \leqslant-k \mathrm{~V}(\mathrm{t}, \mathrm{x})
$$

Thus we can pick a positive and differentiable function $m$ such that

$$
D_{c}^{\alpha, \rho} V(t, x)=-k V(t, x)-m(t) .
$$

Now using the Lemma 2.7, we obtain the following relationship

$$
V(t)=V\left(t_{0}\right) E_{\alpha}\left(-k\left(\frac{t^{\rho}-t_{0}^{\rho}}{\rho}\right)^{\alpha}\right)-\int_{t_{0}}^{t}\left(\frac{t^{\rho}-s^{\rho}}{\rho}\right)^{\alpha-1} E_{\alpha, \alpha}\left(\lambda\left(\frac{t^{\rho}-t_{0}^{\rho}}{\rho}\right)^{\alpha}\right) m(s) \frac{d s}{s^{1-\rho}} .
$$

Neglecting the second term due to its positive, it follows from the fact the function $V$ is positive definite that

$$
V(t, x(t)) \leqslant V\left(t_{0}\right) E_{\alpha}\left(-k\left(\frac{t^{\rho}-t_{0}^{\rho}}{\rho}\right)^{\alpha}\right) .
$$

We get under first assumption the following relationship

$$
\|x(t)\| \leqslant\left\{v\left(t_{0}\right) E_{\alpha}\left(-k\left(\frac{t^{\rho}-t_{0}^{\rho}}{\rho}\right)^{\alpha}\right)\right\}^{\frac{1}{\alpha}} .
$$

We conclude the trivial solution of the generalized fractional differential equation (3.1) is generalized Mittag-Leffler stable.

Many Lyapunov characterizations can be obtained from Theorems 3.3 and 3.4. Here we give the Lyapunov characterizations of the generalized asymptotic stability and the generalized Mittag-Leffler stability for the generalized fractional differential equations. For more information in the Lyapunov characterizations and stability notions, see $[6,23]$. 


\section{Stability analysis of the perturbed generalized fractional differential equations}

In this section, we focus the stability conditions of the trivial solution of the perturbed generalized fractional differential equation defined as

$$
D_{c}^{\alpha, \rho} x=A x+g(t, x)
$$

where $x \in \mathbb{R}^{n}$ is a state variable, $A$ is a matrix in $\mathbb{R}^{n \times n}, g: \mathbb{R}^{+} \times \mathbb{R} \rightarrow \mathbb{R}^{n}$ is a continuous locally Lipschitz function and satisfies the condition $g(t, 0)=0$. We will use the generalized Gronwall lemma. We make the following theorem.

Theorem 4.1. If the matrix $A$ satisfies the condition $|\arg (\lambda(A))|>\frac{\alpha \pi}{2}$, and in addition the condition $\|g(t, x)\|<$ $\gamma(\|x\|)$ is held, then the generalized fractional differential equation (4.1) is generalized asymptotically stable.

The importance of this theorem is the proof. We use the generalized Gronwall lemma described in the Lemma 2.8.

Proof. Firstly, we determine the analytical solution of the generalized fractional differential equation defined by (4.1). We use the Lemma 2.7

$$
x(t)=\eta E_{\alpha}\left(A\left(\frac{t^{\rho}-t_{0}^{\rho}}{\rho}\right)^{\alpha}\right)+\int_{t_{0}}^{t}\left(\frac{t^{\rho}-s^{\rho}}{\rho}\right)^{\alpha-1} E_{\alpha, \alpha}\left(A\left(\frac{t^{\rho}-t_{0}^{\rho}}{\rho}\right)^{\alpha}\right) g(s, x(s)) \frac{d s}{s^{1-\rho}} .
$$

Under the assumption $\|g(t, x)\|<\gamma(\|x\|)$, we have the following relationship

$$
\|x(t)\| \leqslant \eta\left\|E_{\alpha}\left(A\left(\frac{t^{\rho}-t_{0}^{\rho}}{\rho}\right)^{\alpha}\right)\right\|+\gamma \int_{t_{0}}^{t}\left\|\left(\frac{t^{\rho}-s^{\rho}}{\rho}\right)^{\alpha-1} E_{\alpha, \alpha}\left(A\left(\frac{t^{\rho}-t_{0}^{\rho}}{\rho}\right)^{\alpha}\right) s^{\rho-1}\right\|\|x(s)\| d s .
$$

We use the generalized Gronwall Lemma 2.8, we obtain the following relation

$$
\|x(t)\| \leqslant \eta E_{\alpha}\left(A\left(\frac{t^{\rho}-t_{0}^{\rho}}{\rho}\right)^{\alpha}\right) E_{\alpha}\left\{\gamma \Gamma(\alpha) E_{\alpha, \alpha}\left(A\left(\frac{t^{\rho}-t_{0}^{\rho}}{\rho}\right)^{\alpha}\right)\left(\frac{t^{\rho}-t_{0}^{\rho}}{\rho}\right)^{\alpha}\right\} .
$$

Finally, we get the following relationship

$$
\|x(t)\| \leqslant \eta\left\|E_{\alpha}\left(A\left(\frac{t^{\rho}-t_{0}^{\rho}}{\rho}\right)^{\alpha}\right)\right\|\left\|E_{\alpha}\left\{\gamma \Gamma(\alpha) E_{\alpha, \alpha}\left(A\left(\frac{t^{\rho}-t_{0}^{\rho}}{\rho}\right)^{\alpha}\right)\left(\frac{t^{\rho}-t_{0}^{\rho}}{\rho}\right)^{\alpha}\right\}\right\| .
$$

Recalling the condition $|\arg (\lambda(A))|>\frac{\alpha \pi}{2}$, then we obtain $\lim _{t \rightarrow+\infty}\|x(t)\|=0$. Thus, the generalized fractional differential equation (4.1) is generalized asymptotically stable.

The last theorem of this section is an application of the Lemma 2.5. Furthermore, with this next theorem, we give a bound of the Lipschitz constant $\gamma$ under which we do not lose the generalized asymptotic stability of the generalized fractional differential equation (4.1). For that, we define the following theorem.

Theorem 4.2. If the matrix $A$ satisfies the condition that $|\arg (\lambda(A))|>\frac{\alpha \pi}{2}$ and in addition the condition $\|g(t, x)\|<\gamma(\|x\|)$ is held, furthermore if $\lambda_{\min }(\mathrm{Q})-2 \lambda_{\min }(\mathrm{P}) \gamma>0$, then the generalized fractional differential equation (4.1) is generalized asymptotically stable.

Proof. Let the function $\mathrm{V}(\mathrm{x})=\mathrm{x}^{\top} \mathrm{P} x$ be a Lyapunov candidate function for the generalized fractional differential equation (4.1). The objective is to use Lemma 2.5. We calculate the generalized Caputo fractional derivative of $\mathrm{V}$ along the trajectories of equation (4.1), and then we obtain that

$$
D_{c}^{\alpha, \rho} V(x)=D_{c}^{\alpha, \rho}\left(x^{\top} P x\right) \leqslant 2 x^{\top} P_{c^{\alpha}}^{\alpha, \rho} x=(A x+g(t, x))^{\top} P x+x^{\top} P(A x+g(t, x))
$$




$$
\begin{aligned}
& =x^{\top}\left(A^{\top} P+P A\right) x+2 g^{\top}(t, x) \\
& \leqslant-\lambda_{\min }(Q)\|x\|^{2}+2 \lambda_{\min }(P) \gamma\|x\|^{2} \\
& \leqslant-\left[\lambda_{\min }(Q)-2 \lambda_{\min }(P) \gamma\right]\|x\|^{2} .
\end{aligned}
$$

It follows from assumption that $\lambda_{\min }(\mathrm{Q})-2 \lambda_{\min }(\mathrm{P}) \gamma>0$, the generalized fractional differential equation (4.1) is generalized asymptotically stable.

\section{Fractional input stability for the generalized fractional differential equations}

In this section, we investigate the fractional input stability related to a particular class of the generalized fractional differential equations. Generally, the generalized fractional differential equations under consideration is defined by the following equation

$$
\mathrm{D}_{\mathrm{c}}^{\alpha, \rho} x=\mathrm{A} x+\mathrm{Bu},
$$

where $x \in \mathbb{R}^{n}$ is a state variable, $A$ is a matrix in $\mathbb{R}^{n \times n}$ satisfying in addition the condition that $|\arg (\lambda(A))|>\frac{\alpha \pi}{2}, B$ is a matrix in $\mathbb{R}^{n \times m}$ and $u \in \mathbb{R}^{m}$ represents the exogenous input. The main objective is to prove that a converging input for (5.1) generates a converging state and a bounded input for (5.1) also generates a bounded state. In other words that means the generalized fractional differential equation (5.1) is generalized fractional input stable [18].

We recall the definition of the generalized fractional input stability for the generalized fractional differential equations with exogenous inputs.

Definition 5.1 ([18]). The generalized fractional differential equation (5.1) is said to be generalized fractional input stable if, for any input $u \in \mathbb{R}^{m}$, there exist a class $\mathcal{K} \mathcal{L}$ function $\beta$ and a $\mathcal{K}_{\infty}$ function $\gamma$, such that for any initial condition $\eta$, its solution satisfies

$$
\left\|x\left(t, x_{0}, u\right)\right\| \leqslant \beta\left(\|\eta\|, t^{\rho}-t_{0}^{\rho}\right)+\gamma\left(\|u\|_{\infty}\right) .
$$

The fractional input stability can be proved by using the trajectory, but this issue is not all time possible. There exists the generalized fractional differential equations which getting there solutions are not trivial. The alternative is to use the Lyapunov characterizations.

In this section, we use the trajectories and the Lemma 2.7 to prove the generalized fractional input stability. The analytical solution of (5.1) is given by

$$
x(t)=\eta E_{\alpha}\left(A\left(\frac{t^{\rho}-t_{0}^{\rho}}{\rho}\right)^{\alpha}\right)+\int_{t_{0}}^{t}\left(\frac{t^{\rho}-s^{\rho}}{\rho}\right)^{\alpha-1} E_{\alpha, \alpha}\left(A\left(\frac{t^{\rho}-t_{0}^{\rho}}{\rho}\right)^{\alpha}\right) B u(s) \frac{d s}{s^{1-\rho}} .
$$

Applying the euclidean norm on the above expression, we get that

$$
\|x(t)\| \leqslant \eta\left\|E_{\alpha}\left(A\left(\frac{t^{\rho}-t_{0}^{\rho}}{\rho}\right)^{\alpha}\right)\right\|+\|B\|\|u\| \int_{t_{0}}^{t}\left\|\left(\frac{t^{\rho}-s^{\rho}}{\rho}\right)^{\alpha-1} E_{\alpha, \alpha}\left(A\left(\frac{t^{\rho}-t_{0}^{\rho}}{\rho}\right)^{\alpha}\right) s^{\rho-1}\right\| d s .
$$

Note that if the matrix $A$ satisfies the condition $|\arg (\lambda(A))|>\frac{\alpha \pi}{2}$ then there exist a positive constant $M$ such that

$$
\int_{t_{0}}^{t}\left\|\left(\frac{t^{\rho}-s^{\rho}}{\rho}\right)^{\alpha-1} E_{\alpha, \alpha}\left(A\left(\frac{t^{\rho}-t_{0}^{\rho}}{\rho}\right)^{\alpha}\right) s^{1-\rho}\right\| d s \leqslant M .
$$

Thus we obtain the following relationship

$$
\|x(t)\| \leqslant \eta\left\|E_{\alpha}\left(A\left(\frac{t^{\rho}-t_{0}^{\rho}}{\rho}\right)^{\alpha}\right)\right\|+\|B\|\|u\| M .
$$


Let $\beta\left(\left\|x\left(t_{0}\right)\right\|, t^{\rho}-t_{0}^{\rho}\right)=\eta\left\|E_{\alpha}\left(A\left(\frac{t^{\rho}-t_{0}^{\rho}}{\rho}\right)^{\alpha}\right)\right\| \in \mathcal{K L}$, and $\gamma\left(\|u\|_{\infty}\right)=\|B\|\|u\| M \in \mathcal{K}_{\infty}$. Finally, we obtain

$$
\left\|x\left(t, x_{0}, u\right)\right\| \leqslant \beta\left(\left\|x\left(t_{0}\right)\right\|, t^{\rho}-t_{0}^{\rho}\right)+\gamma\left(\|u\|_{\infty}\right) .
$$

Thus, the generalized fractional differential equation (5.1) is generalized fractional input stable. We have briefed the fractional input stability in the context of the generalized fractional differential equations. All results existing with this new stability notion can be applied on the generalized fractional differential equations with exogenous inputs. For more main results related to the fractional input stability and the Mittag-Leffler input stability see [18] and in integer order context in [22].

We recall the Lyapunov characterization of the generalized fractional input stability, the proof can be found in [18].

Theorem 5.2. Let there exist a positive function $\mathrm{V}: \mathbb{R}^{+} \times \mathbb{R}^{\mathrm{n}} \longrightarrow \mathbb{R}$ which is continuous and differentiable, and the class $\mathcal{K}_{\infty}$ functions $\chi_{1}, \chi_{2}$ and class $\mathcal{K}$ functions $\chi_{3}, \chi_{4}$ satisfying the following conditions:

1. $\chi_{1}(\|x\|) \leqslant V(t, x) \leqslant \chi_{2}(\|x\|)$;

2. If for any $\|x\| \geqslant \chi_{4}((|u|)) \Longrightarrow D_{\mathcal{c}}^{\alpha, \rho} V(t, x) \leqslant-\chi_{3}((\|x\|))$.

Then the generalized fractional differential equation (5.1) is generalized fractional input stable.

\section{Applications and numerical examples}

In this section, we study some examples. We investigate of applying the main results of the paper: the generalized asymptotic stability, the generalized fractional input stability, and the application of the Lemma 2.5.

Let the generalized fractional differential equation with exogenous input defined by

$$
\left\{\begin{array}{l}
D_{c}^{\alpha, \rho} x_{1}=-x_{1}+x_{2} \\
D_{c}^{\alpha, \rho} x_{2}=-x_{2}+u
\end{array}\right.
$$

where $x=\left(x_{1}, x_{2}\right) \in \mathbb{R}^{2}$ and $u \in \mathbb{R}$ represents the exogenous input. We use the Lyapunov direct method of studying the generalized fractional input stability, see in [18]. To reach our end, we consider the Lyapunov candidate function defined by $V(x)=\frac{1}{2}\left(x_{1}^{2}+x_{2}^{2}\right)=\frac{\|x\|^{2}}{2}$. The first step consists of calculating the Caputo generalized fractional derivative of the Lyapunov function along the trajectories; it yields that

$$
\begin{aligned}
\mathrm{D}_{\mathrm{c}}^{\alpha, \rho} \mathrm{V}(\mathrm{t}, \mathrm{x})=\mathrm{D}_{\mathrm{c}}^{\alpha, \rho}\left(\mathrm{x}^{\top} \mathrm{P} x\right) \leqslant 2 x^{\top} \mathrm{PD}_{\mathrm{c}}^{\alpha, \rho} x & =-x_{1}^{2}+x_{1} x_{2}-x_{2}^{2}+x_{2} \mathrm{u} \\
& =-\frac{1}{2} x_{1}^{2}+\frac{1}{2} \chi_{2}^{2}-x_{2}^{2}+\frac{1}{2} \chi_{2}^{2}+\frac{1}{2}\|\mathrm{u}\|^{2} \\
& \leqslant-\frac{1}{2} x_{1}^{2}+\frac{1}{2}\|\mathrm{u}\|^{2} \\
& \leqslant-\frac{1}{2}(1-\theta) x_{1}^{2}-\frac{1}{2} \theta x_{1}^{2}+\frac{1}{2}\|u\|^{2}
\end{aligned}
$$

with $\theta \in(0,1)$. If $\|x\| \geqslant \frac{\|u\|}{\theta}$ it implies that $D_{c}^{\alpha, \rho} V(t, x) \leqslant-\frac{1}{2}(1-\theta) x_{1}^{2}$. Then from Theorem 5.2, the generalized fractional differential equation (6.1) is generalized fractional input stable.

The author remark is that, we use the synchronization or the stabilization to study the stability of the generalized fractional differential equations. But now one can also use the generalized fractional input stability to analyze the stability of the generalized fractional differential equations with exogenous inputs. One can use this new stability notion to study the stability of the fractional neural network, and many other categories of the fractional differential equations with inputs. For more information on this new stability notion, see $[18,19]$. 
Let the generalized fractional differential equation with perturbation term defined by

$$
\mathrm{D}_{\mathrm{c}}^{\alpha, \rho} x=-x+\frac{1}{2} \sin x .
$$

As in the previous example we use the Lyapunov direct method to prove the generalized MittagLeffler stability. For that we take the Lyapunov function defined by $V(x)=\frac{\|x\|^{2}}{2}$. The Caputo generalized fractional derivative of $\mathrm{V}$ along the trajectories yields that

$$
\mathrm{D}_{\mathrm{c}}^{\alpha, \rho} \mathrm{V}(\mathrm{t}, \mathrm{x})=\mathrm{D}_{\mathrm{c}}^{\alpha, \rho}\left(\mathrm{x}^{\top} \mathrm{P} x\right) \leqslant 2 x^{\top} \mathrm{PD}_{\mathrm{c}}^{\alpha, \rho} \chi=\chi\left(-x+\frac{1}{2} \sin x\right)=-\chi^{2}+\frac{1}{2} x \sin x .
$$

We know that $g(t, x)=\sin x$ is Lipschitz and the Lipschitz constant $\gamma=1$ verifies the assumption related to the function $\mathrm{g}$ in the Theorem 4.2. Thus, we obtain that

$$
\mathrm{D}_{\mathrm{c}}^{\alpha, \rho} \mathrm{V}(\mathrm{t}, \mathrm{x})=\mathrm{D}_{\mathrm{c}}^{\alpha, \rho}\left(\mathrm{x}^{\top} \mathrm{P} x\right) \leqslant 2 \mathrm{x}^{\top} \mathrm{PD}_{\mathrm{c}}^{\alpha, \rho} \mathrm{x}=-\mathrm{x}^{2}+\frac{1}{2} \mathrm{x} \sin \mathrm{x}=-\mathrm{x}^{2}+\frac{1}{2} \chi^{2}=-\frac{1}{2} \mathrm{~V}(x, \mathrm{t})
$$

Thus, under the Theorem 4.2, the generalized fractional differential equation (6.2) is generalized MittagLeffler stable.

We finish this section by the generalized asymptotic stability of the generalized fractional Lorenz equation defined as

$$
\left\{\begin{array}{l}
D_{c}^{\alpha, \rho} x_{1}=\sigma\left(y_{1}-x_{1}\right) \\
D_{c}^{\alpha, \rho} y_{1}=\rho x_{1}-y_{1}-x_{1} z_{1} \\
D_{c}^{\alpha, \rho} z_{1}=-\beta z_{1}+x_{1} y_{1}
\end{array}\right.
$$

where $x=\left(x_{1}, y_{1}, z_{1}\right) \in \mathbb{R}^{3}$ and $\sigma, \rho, \beta$ are positive constants. We notice the generalized fractional Lorentz equation can be rewritten as follows

$$
\left(\begin{array}{l}
D_{c}^{\alpha, \rho} x_{1} \\
D_{c}^{\alpha, \rho} y_{1} \\
D_{c}^{\alpha, \rho} z_{1}
\end{array}\right)=\left(\begin{array}{ccc}
-\sigma & \sigma & 0 \\
\rho & -1 & 0 \\
0 & 0 & -\beta
\end{array}\right)\left(\begin{array}{l}
x_{1} \\
y_{1} \\
z_{1}
\end{array}\right)+\left(\begin{array}{c}
0 \\
-x_{1} y_{1} \\
x_{1} y_{1}
\end{array}\right)
$$

And then the fractional generalized differential equation (6.3) can be expressed in the form $D_{\mathcal{c}}^{\alpha, \rho} \chi=$ $A x+g(t, x)$. We have the matrix and the function defined as follows

$$
A=\left(\begin{array}{ccc}
-\sigma & \sigma & 0 \\
\rho & -1 & 0 \\
0 & 0 & -\beta
\end{array}\right), \quad g(t, x)=\left(\begin{array}{c}
0 \\
-x_{1} y_{1} \\
x_{1} y_{1}
\end{array}\right) .
$$

To prove the generalized asymptotic stability for particular Lorenz differential equation, we have to verify the assumptions in the Theorem 4.2. The eigenvalues of the matrix $A$ are given by

$$
\lambda_{1}=\frac{-\sigma-1-\sqrt{(\sigma-1)^{2}+4 \sigma \rho}}{2}, \quad \lambda_{2}=\frac{-\sigma-1+\sqrt{(\sigma-1)^{2}+4 \sigma \rho}}{2}, \quad \lambda_{3}=-\beta .
$$

It's straightforward to see the matrix $A$ satisfies the condition $|\arg (\lambda(A))|>\frac{\alpha \pi}{2}$ when $0<\rho<1$. Furthermore we have $\|g(t, x)\|=\sqrt{x_{1}^{2}\left(y_{1}^{2}+z_{1}^{2}\right)}<\|x\|^{2}$ with $\gamma=1$. All assumptions of the Theorem 4.2 are held. Then the generalized fractional differential equation defined by (6.3) is generalized asymptotically stable.

\section{Conclusion}

In this paper, we have analyzed the generalized asymptotic stability, the generalized Mittag-Leffler stability of the generalized fractional differential equations. We have introduced the new stability notion known as the fractional input stability in the context of the generalized fractional differential equations. A useful Lemma helping to study the generalized stability notions with the Lyapunov direct method is also provided in this paper. 


\section{References}

[1] Y. Adjabi, F. Jarad, T. Abdeljawad, On generalized fractional operators and a Gronwall type inequality with applications, Filomat, 31 (2017), 5457-5473. 1, 2.1, 2.2, 2.3, 2.4, 2, 2, 2.8

[2] A. Atangana, D. Baleanu, New fractional derivatives with nonlocal and non-singular kernel: theory and application to heat transfer model, arXiv, 2016 (2016), 8 pages. 1

[3] A. Atangana, I. Koca, New direction in fractional differentiation, Math. Nat. Sci., 1 (2017), 18-25. 1

[4] D. Baleanu, A. K. Golmankhaneh, A. K. Golmankhaneh, The dual action of fractional multi time hamilton equations, Int. J. Theor. Phys., 48 (2009), 2558-2569. 1

[5] D. Baleanu, G.-C. Wu, S.-D. Zeng, Chaos analysis and asymptotic stability of generalized caputo fractional differential equations, Chaos Solitons Fractals, 102 (2017), 99-105. 1

[6] A. Ben Makhlouf, Stability with respect to part of the variables of nonlinear Caputo fractional differential equations, Math. Commun., 23 (2018), 119-126. 3

[7] N. A. Camacho, M. A. D. Mermoud, J. A. Gallegos, Lyapunov functions for fractional order systems, Commun. Nonli. Sci. Numer. Simulat., 19 (2014), 2951-2957. 2

[8] F. Jarad, T. Abdeljawad, A modified laplace transform for certain generalized fractional operators, Results Nonlinear Anal., 2 (2018), 88-98. 2, 2.7, 2

[9] F. Jarad, T. Abdeljawad, D. Baleanu, On the generalized fractional derivatives and their caputo modification, J. Nonlinear Sci. Appl., 10 (2017), 2607-2619. 1

[10] F. Jarad, E. Uğurlu, T. Abdeljawad, D. Baleanu, On a new class of fractional operators, Adv. Difference Equ., 1 (2017), 16 pages. $1,2.3$

[11] U. N. Katugampola, New approach to a generalized fractional integral, Appl. Math. Comput., 218 (2011), 860-865. 1, 2.1, 2.2, 2.3

[12] U. N. Katugampola, A new approach to generalized fractional derivatives, Bull. Math. Anal. Appl., 6 (2014), 1-15. 1

[13] Y. Li, Y. Q. Chen, I. Podlubny, Mittagleffler stability of fractional order nonlinear dynamic systems, Automatica, 45 (2009), 1965-1969. 1, 2

[14] S. Priyadharsini, Stability of fractional neutral and integrodifferential systems, J. Fract. Calc. Appl., 7 (2016), 87-102. 1, 2

[15] N. Sene, Exponential form for lyapunov function and stability analysis of the fractional differential equations, J. Math. Computer Sci., 18 (2018), 388-397. 2.4, 3, 3

[16] N. Sene, Lyapunov characterization of the fractional nonlinear systems with exogenous input, Fractal Fract., 2 (2018), 10 pages. 1,2

[17] N. Sene, Analytical solutions of Hristov diffusion equations with non-singular fractional derivatives, Chaos, 29 (2019), 11 pages. 2

[18] N. Sene, Fractional input stability and its application to neural network, Discrete Contin. Dyn. Syst. Ser. S, 13 (2019), 853-865. 5, 5.1, 5, 6

[19] N. Sene, Fractional input stability for electrical circuits described by the Riemann-Liouville and the Caputo fractional derivatives, AIMS Math., 4 (2019), 147-165. 6

[20] N. Sene, On stability analysis of the fractional nonlinear systems with hurwitz state matrix, J. Fract. Calc. Appl., 10 (2019), 1-9.

[21] N. Sene, Solution of fractional diffusion equations and Cattaneo-Hristov diffusion model, Int. J. Anal. Appl., 17 (2019), 191-207. 1

[22] E. D. Sontag, Y. Wang, On characterizations of the input-to-state stability property, Systems Control Lett., 24 (1995), 351-359. 5

[23] A. Souahi, A. Ben Makhlouf, M. A. Hammami, Stability analysis of conformable fractional-order nonlinear systems, Indag. Math. (N. S.), 28 (2017), 1265-1274. 1, 3

[24] H. P. Ye, J. M. Gao, Y. S. Ding, A generalized gronwall inequality and its application to a fractional differential equation, J. Math. Anal. Appl., 328 (2007), 1075-1081. 1

[25] F. R. Zhang, G. R. Chen, C. P. Li, J. Kurths, Chaos synchronization in fractional differential systems, Philos. Trans. R. Soc. Lond. Ser. A Math. Phys. Eng. Sci., 371 (2013), 26 pages. 1 